\title{
REAL-TIME P2P STREAMING BASED ON PLAYBACK-RATE IN MANETS
}

\author{
Chia-Cheng $\mathrm{Hu}^{1,2, *}$, Zhong-bao Liu ${ }^{2,3}$, Hong-Bo Zhou ${ }^{2}$ and Chong- \\ JieZhang ${ }^{2}$ \\ ${ }^{1}$ College of Information Engineering, Yango University, Fuzhou, China \\ ${ }^{2}$ School of Software, Quanzhou University of Information Engineering, \\ Quanzhou, China \\ ${ }^{3}$ School of Software, North University of China, Taiyuan, China \\ ${ }^{*}$ Corresponding author
}

\begin{abstract}
In a QoS-intensive multimedia application, Media-on-Demand (MoD) streaming can be delivered to asynchronous users with asynchronous requirement of MoD and VCR-like operation support. It is a critical challenge to propose a segment scheduling algorithm for real-time Peer to Peer (P2P) streaming services in mobile ad hoc networks (MANETs).However, it is a big challenge to provide MOD multimedia streaming to a large population of clients due to the asynchronous users. In this paper, we propose areal-time $P 2 P$ scheduling algorithm by scheduling the segments evenly transmitted into the network according to the playback-rate of the real-time streaming service. The proposed algorithm schedules the segments from the peer with less bandwidth consumption to the network for further saving the limited bandwidth. On the other hand, it is adaptive to host mobility. Extensive simulations illustrate the effectiveness of the proposed scheme.
\end{abstract}

\section{KEYWORDS}

Mobile Ad Hoc Networks, Playback Rate, Segment Scheduling and Timely P2P Streaming

\section{INTRODUCTION}

In a QoS-intensive multimedia application, Media-on-Demand (MoD) streaming on Internet can be delivered to asynchronous users with asynchronous requirement of MoD and VCR-like operation support. However, it is a big challenge to provide MOD multimedia streaming to a large population of clients due to the asynchronous users and the limited provider capacity. To tackle the issue, peer-to-peer ( $\mathrm{P} 2 \mathrm{P})$ networks has emerged as a powerful and popular paradigm for many scalable problems over Internet. The basic design philosophy of P2P networks is to encourage users to act as both clients and servers, namely as peers. In a P2P network, the peers collaborate for the purpose of providing streaming service to each other in the sense that they can all behave as clients and servers. Since a peer downloads some segments for its own playing and then caches them to serve future requests from the other peers, the segments (which constitute the multimedia file) can be spread out quickly among the peers. A mobile ad-hoc network (MANET) is a kind of networks, and it is composed of mobile devices that can arrange themselves in various ways and operate without strict top-down network administration. Nearly, P2P streaming applied in MANETs has become a new focus in the P2P research field [1-4]. Real-time streaming is a necessary requirement for viewing multimedia files, in which each segment should be received 
before its playback deadline. Today, several works [5-14]have been proposed for P2P applications in MANETs. However, these works do not focus mainly on the scheduling scheme, and they cannot achieve a timely delivery. In this paper, we focus on segment scheduling and aim to propose a delay-sensitive segment scheduling algorithm for real-time $\mathrm{P} 2 \mathrm{P}$ streaming applications in MANETs. In the proposed algorithm, we adopt a distinct strategy of using the limited bandwidth in MANETs more efficiently by a rate control mechanism, which determines the number of segments transmitted into the network according to the playback-rate of the requested media file. By the aid of the rate control mechanism, the bandwidth consumption and large buffer size problems can be alleviated significantly. On the other hand in order to satisfy the real-time requirement of the MoD multimedia file, the segments with approaching play-deadlines are scheduled with high priorities. Since a segment may be cached at multiple peers in a P2P system, it will schedule the segment to be transmitted from the peer with less bandwidth consumption to the network. In the next section, the previous P2P scheduling algorithms in MANETs are first reviewed. The detailed design of proposed scheduling algorithms is given in Section 3. In Section 4, the performance evaluation is carried out. Finally, this paper concludes with some remarks in Section 5.

\section{RELATED WORKS}

Recently, several P2P scheduling algorithms have been proposed in MANETs [5-14]. Reference [5] achieves this goal by a layered coding method for streaming a multimedia object between two mobile peers. The method divides a video segment into a base layer and several enhancement layers. The base layer has a lower bit rate compared to the original stream. It can be decoded independently to reconstruct the original video stream with a lower resolution or frame rate. However, main challenge brought on by adopting layered coding method in P2P streaming is a need for more complex scheduling algorithms. Whereas a scheduling algorithm for non-layered streaming only is concerned with throughput maximization, scheduling layered streaming has to take other constraints into account. For example, scheduling a higher layer to be delivered in addition to a lower layer may render a vain transmission if packet loss occurs in the lower layer.

In [6], the V3 architecture for live video streaming is a cooperative streaming architecture among moving vehicles. It incorporates a signaling mechanism to continuously trigger video sources to send video data back to receivers. Broadcasting and multicasting have also been used for streaming media services to mobile users [7, 8]. They are bandwidth efficient transmission mechanisms for applications where there are multiple participants. With the services, applications send one copy of the information from the provider to the receivers. However, the receivers are required to be connected and tuned at the right channel of broadcasting or multicasting. Repeated broadcasting or multicasting is hard to solve the issue of asynchronous users due to asynchronous requirement of the same media at different times and locations.

In [9], the paper proposes a middleware that is adapted to the characteristics of the wireless medium and resource restrictions of mobile nodes. It provides secure access to the stored information for the operations of demanding applications such as multimedia and cooperative services. In [10], the paper studies the performance effects of caching derived from the of different wireless MAC layers, such as 802.11-based ad hoc networks and multi-interfacemultichannel-based mesh networks. Then based on the results, it proposes an asymmetric approach to identify the best nodes to cache the data. In [11], the authors propose a protocol for wireless $\mathrm{P} 2 \mathrm{P}$ resource sharing. The idea is to make use of locality by assigning peers, which are close in the physical network.

In [12], the authors propose a distributed algorithm for scheduling the multiple senders for multisource transmission in mobile P2P networks. The proposed algorithm aims to maximize the data 
rate and minimize the power consumption. In [13], the system takes advantage of node mobility by designating stable nodes as community coordinators for file searching. In [14], the authors propose a new concept of data file replication, which considers both node storage and meeting frequency. Then, a distributed data file replication protocol is proposed to implement the concept. However, these works [13-20] do not focus mainly on the data scheduling scheme, and they cannot achieve a timely delivery.

\section{DELAY-SENSITIVE SEGMENT SCHEDULING ALGORITHM}

In this section, we propose a delay-sensitive segment scheduling algorithm for real-time P2P streaming applications in MANETs. The lookup function is provided by P2P lookup protocols to collect the information of the segments cached on potential peers. Some P2P lookup protocols, such as Chord [15] and Pastry [16], should be extended for the proposed algorithm to enable the lookup result to cover multiple potential peers.

The proposed algorithm adopts a distinct transmission rate control strategy, in which the number of segment s transmitted into the network is decided by the playback-rate of the requested streaming service. The rate control strategy aims to efficiently use the MANET limited bandwidth by scheduling only an enough amount of segments for satisfying the playback-rate of the requested streaming service.

It schedules the segments with approaching play-deadlines first in order to satisfying the real-time requirement of the MoD multimedia file. Based on this consideration, the priority of a segment is computed as the inverse of the period from the current time to the playback deadline of this segment. DSSSA schedules the segments in the following sequence. First, it schedules the segments whose play-deadlines are approaching. These segments are called as urgent segments. Second, it schedules more segments by selecting those with higher priorities for satisfying the playback-rate of the requested streaming service. Third, it schedules the segments, which are only available at few peers, in order to disseminate segments quickly. These segments are defined as scare segments, if they are available only at not more than $\gamma$ peers, where $\gamma>1$ is a predefined integer.

Since a segment may be cached at multiple peers in a P2P system, the proposed algorithm will schedule the segment to be transmitted from the peer with less bandwidth consumption to the network. In order to achieve the purpose, it selects the route with minimal total medium time to the network. The total medium time of a route is to sum the end-to-end delay of the route and the blocking time on all the neighbors of the forwarders along the route. In MANETs, when a host is transmitting packets, its neighbors are blocked since it shares the radio channel with its neighbors. A route with minimal total medium time can reduce the bandwidth consumption to the network.

When a new coming peer intends to request P2P streaming service, it first invokes a P2P lookup protocol for obtaining the values of the playback-rate of the requesting streaming service, the playback-rates of the segments, the playback deadlines of the segments, the information of the segments stored in the peers. Further, the delay-sensitive routing protocol of our previous work [17] for obtaining the values of the end-to-end delays and total medium time of the routes from the peers to the new coming peer.

The outlines of the proposed algorithm are as follows: 
Input: (1) the playback-rate of the requesting streaming service,

(2)the playback-rates of the segments,

(3) the playback deadlines of the segments,

(4)the information of the segments stored in the peers,

(5)the end-to-end delays and total medium time of the routes from the peers to the new coming peer.

Output: the schedule of the un-scheduled segments.

Begin

Step 1. Set the priorities of the un-scheduled segments. The priority of a segment is computed as the inverse of the period from the current time to the playback deadline of the segment.

Step2. Determine an unscheduled segment $s$ with the highest priority.

Step3. Determine a peer $p$ with the minimal total medium time among the peers keeping segment $s$.

Step4. Schedule the segment $s$ from the peer $p$.

Step 5. If there are the un-scheduled segments and the rate of transmitting the scheduled segments is smaller than the playback-rate, go to Step 2.

Step 6. If there is the un-scheduled segment $s$ who is available only at not more than $\gamma$ peers, determine a peer $p$ with the minimal total medium time among the peers keeping segment $s$ and schedule the segment $s$ from the peer $p$.

Step 7. If there is the un-scheduled segment $s$ who is available only at not more than $\gamma$ peers,

end go to Step 6.

If a potential provider peers provides a timely segment transmission from, i.e., the time of receiving the requesting segment exceeds the playback deadline, it stops the segment requesting from the requesting peer. Once a schedule for satisfying the playback-rate of the requested streaming service cannot be made from the rest potential provider peers, peers execute the P2P lookup protocol and the delay-sensitive routing protocol again.

\section{Performance Analysis}

In this section, we illustrate the performance of our proposed algorithm by simulation examples. Simulations are implemented using the Network Simulator 2 package (ns-2, version 2.29) [18].In the simulations, our proposed algorithm uses Chord [15] as the P2P lookup protocol, and use the delay-sensitive routing protocol of our previous work [17] as the routing protocol. In the simulation environment, 50 hosts are randomly distributed over a $1000 \mathrm{~m} \times 1000 \mathrm{~m}$ area. The IEEE $802.11 \mathrm{~b}$ is used as our MAC/PHY protocol, i.e., there are four available data rates 1, 2, 5.5, and 11 Mbps. Packets are sent using the un-slotted Carrier Sense Multiple Access with Collision Avoidance (CSMA/CA). Each host has a First-In-First-Out transmission queue of no more than 64 packets at the MAC layer.

The simulations are performed by measuring the following three indices: admission rate, successful receiving rate, and buffer size. Successful receiving rate is the ratio of the number of the segments that are received before their playback deadlines to the number of the requesting segments. Admission rate is the ratio of the number of requested peers admitted to the number of peer requested. When the admission rate goes up, the network capacity increases. In the simulations of Figures 1-3, 50 peers are generated as a Possion process with an arrival rate of 5 requests per minute. Each peer is hosted on each of the 50 hosts. A special video stream is constructed and re-used for all simulation runs. Its stream content is 30 minutes of a movie with an accurate rate-control package to generate a stream with a constant bit-rate of $200 \mathrm{Kbps}$. Each peer is operated according to an On-Off model that simulates peer departing and joining. During 
each 6 minutes time-slot, a peer is on or off with probabilities 0.9 and 0.1 respectively. Also, it could switch off at the beginning of a time-slot for the rest of the run at any time slot with probability 0.05 . In order to simulate the end-game characteristics when peers rapidly depart the P2P overlay at the end of the video stream's transmission, each peer could switch-off with a probability of 0.5 in the last 5 minutes of the run.

Figure 1demonstratesthe admission rates of our proposed algorithm. The results show that our proposed algorithm is effective in using the limited bandwidth of MANETs. The result is derived from its rate control mechanism by only scheduling the enough number of segments to be transmitted into the network for satisfying the playback-rate of the requested media file. In the results of Figure 2, our proposed algorithm obtains small buffer size. Figure 3demonstrates that DSSSA is sensitive in providing timely segment transmission by the aid of our previous work [17] that can estimate the end-to-end delays and determine delay-sensitive routes.

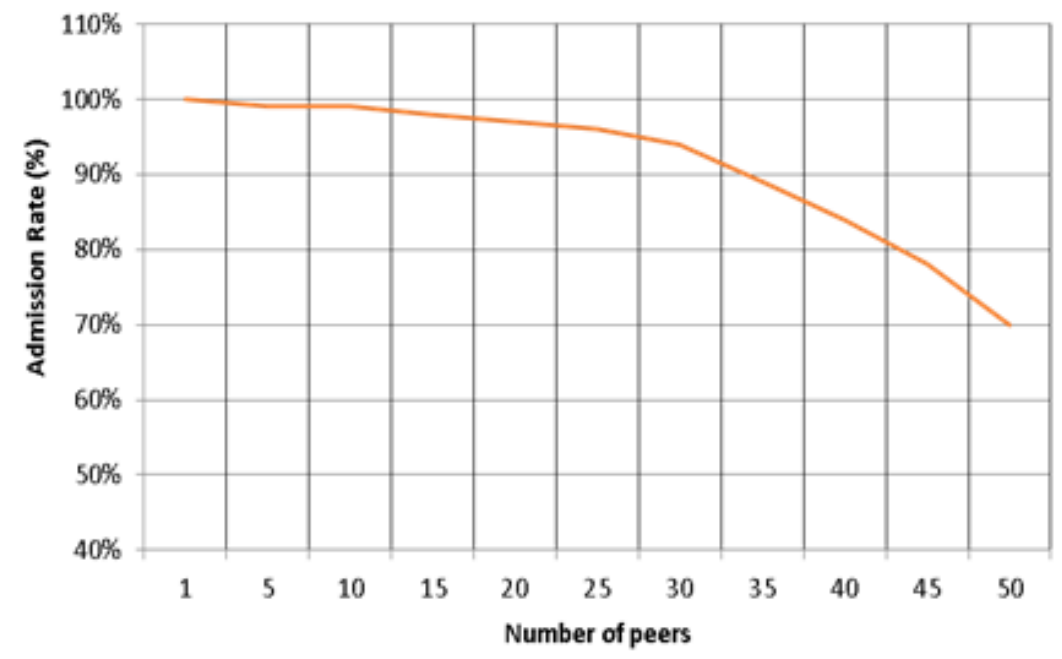

Figure 1. Admission rate.

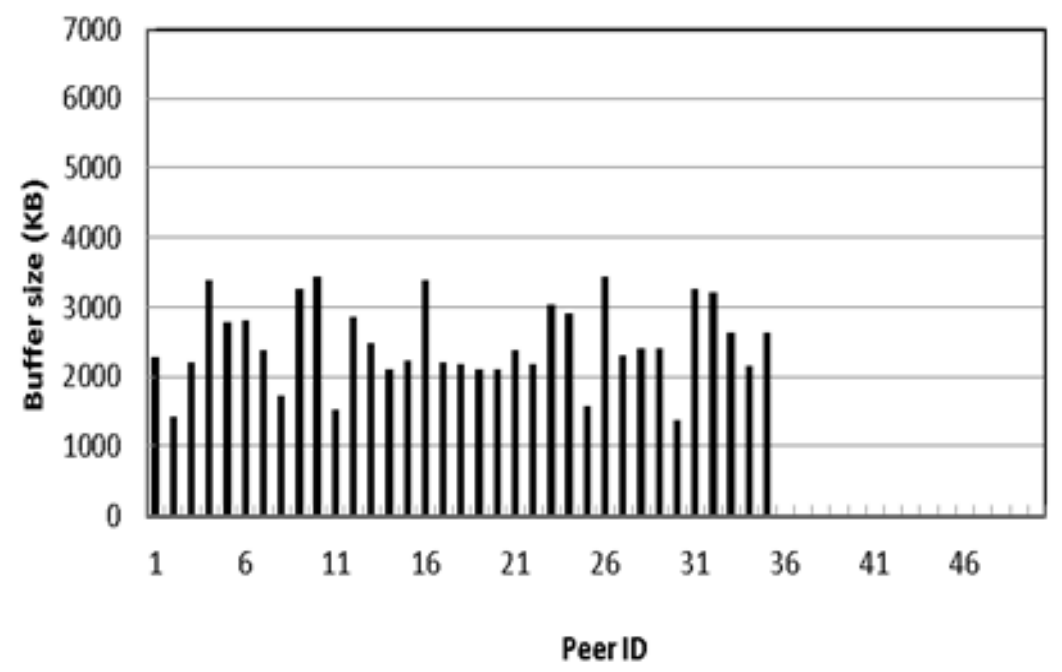

Figure 2. Buffer size.

The simulation environment in Figure 4 is the same as that adopted in Figure 1. But the difference is that the mobility of the 50 hosts is based on the random waypoint model [19], in which a host's 
movement consists of a sequence of random length intervals, called mobility epochs. During each epoch, a host moves in a constant direction and at a constant speed. Their speed varies from 5 to 20 meters per second.

Figure4 compares the successful receiving rates of our proposed algorithm under the assumption of mobile hosts. Compared with the simulation results of Figure 1, our proposed algorithm is adaptive on relieving the mobility problem since it has slightly decreasing of successful receiving rate.

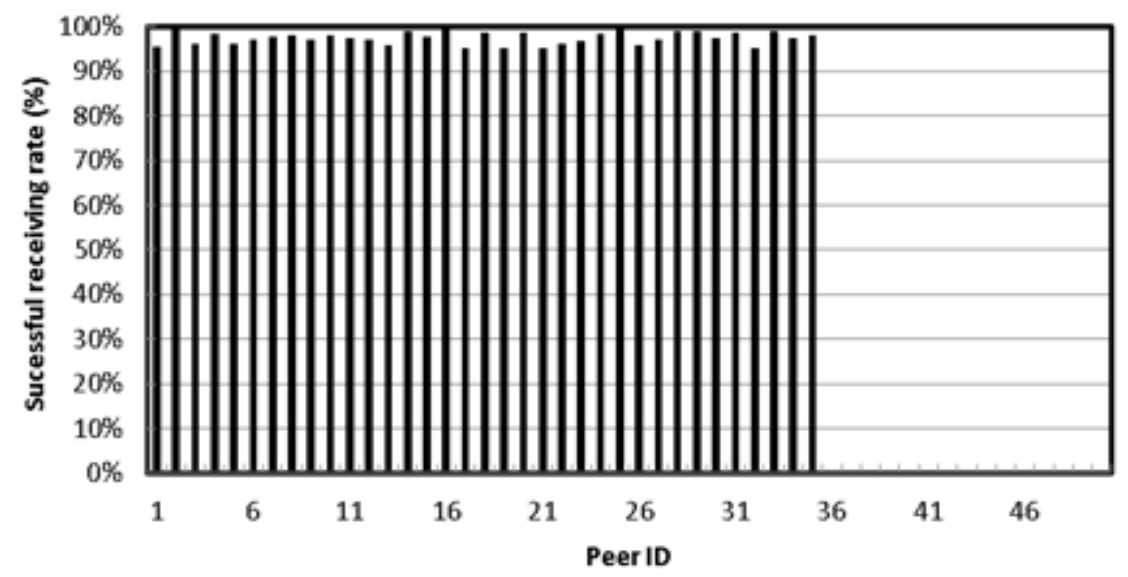

Figure 3. Successful receiving rates.

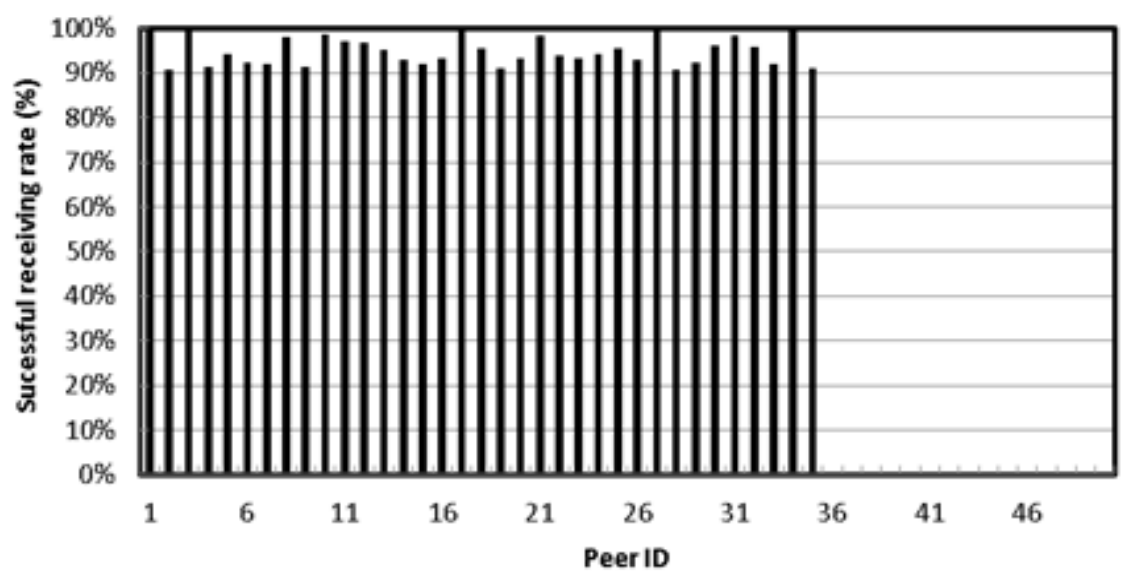

Figure 4. Successful receiving rates under mobile hosts.

\section{CONCLUSIONS}

In order to exploit wireless resource efficiently and provide timely $\mathrm{P} 2 \mathrm{P}$ streaming services in MANETs, we proposed a delay-sensitive segment scheduling algorithm. Taking the playback-rate of the real-time streaming service into consideration, the proposed algorithm schedules the segments of the service evenly transmitted into a MANET. As a consequence of the consideration, the limited bandwidth in the network can be used efficiently. 


\section{ACKNOWLEDGEMENTS}

This work was supported in part by the Fujian Provincial Key Laboratory of Cloud Computing and Internet-of-Thing Technology, China.

\section{REFERENCES}

[1] I. M. Moraes, M. E. M. Campista, L. H. M. K. Costa, O. C. M. B. Duarte, J. L. Duarte, D. G. Passos, C. V. N. de Albuquerque\& M. G. Rubinstein, (2008) "On impact of user mobility on peer-to-peer video streaming,” IEEE Wireless Communication, vol. 15, no. 6, pp. 54-62.

[2] X. F Liao, H. Jin\& W. B. Jiang, (2008) "Moving P2P Live Streaming to Mobile and Ubiquitious Environment,” IEEE. Computing and Informatics, vol. 27, no. 5, pp. 823-835.

[3] Z. J. Chen, C. Lin\& X. G. Wei, (2009) "Enabling on-demand internet video streaming services to multi-terminal users in large scale," IEEE Transactions on Consumer Electronics, vol. 55, no. 4, pp. 1988-1996 .

[4] G. T. Xue, H. Shi, J. Y. You \& W. S. Yao, (2003) "Distributed stable-group differentiated admission control algorithm in mobile peer-to-peer media streaming," Chinese Journal of Electronics, vol. 12, no. 4 , pp. $517-521$.

[5] M. Qin \& R. Zimmermann, (2007) "Improving mobile adhoc streaming performance through adaptive layer selection with scalable video coding," Proc ACM Multimedia.

[6] M. Guo, M. H. Ammar \& E. W. Zengura, (2005) "V3: A vehicle-to-vehicle live video streaming architecture," Pervasive and Mobile Computing, vol. 1, no. 4, pp. 404-424.

[7] H. Jenkac, T. Stockhammer \& W. Xu, (2006) "Asynchronous and reliable on-demand media broadcast," IEEE Network, vol. 20, no. 2, pp. 14-20.

[8] M. F. Leung\& S. H. G. Chan, (2007) "Broadcast-based peer-to-peer collaborative video streaming among mobiles," IEEE Transactions on Broadcasting, vol. 53, no. 1, pp. 350-361.

[9] D. F. Macedo, A. L. dos Santos\& J. M. S. Nogueira, (2009) "A distributed information repository for autonomic context-aware MANETs," IEEE Transactions on Network and Service Management, vol. 6 , no. 1 , pp. $45-55$.

[10] J. Zhao, P. Zhang, G. H. Cao\& C. R. Das, (2010) "Cooperative caching in wireless p2p networks: design, implementation, and evaluation," IEEE Transactions on Parallel and Distributed Systems, vol. 21 , no. 2, pp. 229-241.

[11] C. Canali, M. E. Renda, P. Santi \& S. Burresi, (2010) "Enabling efficient peer-to-peer resource sharing in wireless mesh networks," IEEE Transactions on Mobile Computing, vol. 9, no. 3, pp. 333347.

[12] P. Si, F.R. Yu, H. Ji \& V.C.M. Leung, (2009) "Distributed sender scheduling for multimedia transmission in wireless mobile peer-to-peer networks," IEEE Transactions on Wireless Communications, vol. 8, no. 9, pp. 4594-4603.

[13] K. Chen, H. Shen\& H. Zhang, (2014) "Leveraging social networks for P2P content-based file sharing in disconnected MANETs," IEEE Transactions on Mobile Computing, vol. 13, no. 2, pp. 235-249.

[14] K. Chen \& H. Shen, (2015) "Maximizing P2P file access availability in mobile ad hoc networks though replication for efficient file sharing," IEEE Transactions on Computers, vol. 64, no. 4, 2015, pp. 1029-1042. 
[15] I. Stoic, R. Morris, D. R. Karger, M. F. Kaashoek \& H. Balakrishnan, (2003) "Chord: a scalable peerto-peer lookup protocol for Internet," IEEE Transactions on Networking, vol. 11, no. 9, pp. 17-32.

[16] A. Rowstron \& P. Druschel, (2001) "Pastry: scalable, distributed object location and routing for largescale peer-to-peer systems," Proc. 18th IFIP/ACM Conf. Dist. Sys. Platforms (Middleware 2001), Heidelberg, Germany, pp. 329-350.

[17] C. C. Hu, (2011) "Delay-sensitive routing in multi-rate MANETs," Journal of Parallel and Distributed Computing, vol. 71, pp. 53-61.

[18] Network Simulator (Version 2), http://www-mash.cs.berkeley.edu/ns/.

[19] C.Bettstetter, G. Resta \& P. Santi, (2003) "The node distribution of the random waypoint mobility for wireless ad hoc," IEEE Transactions on Mobile Computing, vol. 2, no. 3, pp. 257-269. 